\title{
Practical Synthesis of Novel Citryl Glycoside, the Component of the Rhizomes of Gastrodia elata
}

\author{
Jung-Hyun Choi and Dong-Ung Lee* \\ Department of Biotechnology: Dongguk University, Gyeongiu 780-714, Korea. 'E-mail: dulee(aldonggukac.kr \\ Received July I, 2008
}

Key Words : Trimethylcitryl- $\beta$ D-galactopyranoside, Practical synthesis, Glycosylation, Deacetylation

The rhizome of Gastrodia elata Blume (Gastrodiae Rhizoma, Orchidaceae) has been used in traditional medicine as an anticonvulsant and sedatives in Korea, Japan and China. 'Identification of its constituents has focused mainly on phenolic compounds: besides a major phenolic glucoside (gastrodin [4-( $\beta$-D-glucopyranosyloxy)benzyl alcohol]). more than 15 phenolics have been isolated. ${ }^{2-5}$ Among them. tris[(4- $\beta$-D-glucopyranosyloxy)benzyl] citrate (parishin). 1,2-bis[(4-( $\beta$-D-glucopyranosyl-oxy)benzyl] citrate (parishin B) and 1,3-bis[(4-( $\beta$-D-glucopyranosyl-oxy) benzyl] citrate (parishin $C$ ) contain a citrate moiety, further. 1.5-dimethylcitrate $^{6}$ was also reported. Recently, we isolated a citrate containing constituent from Gastrodiae Rhizoma, and characterized its structure as trimethylcitryl- $\beta$ D-galactopyranoside. $^{7}$ This new natural citrate glycoside shows an inhibitory activity on GABA transaminase, suggesting an anticonvulsive effect.

We report the practical synthesis of trimethylcitryl- $\beta$-Dgalactopyranoside from citric acid.

The synthetic procedure presented in Figure 1 shows that the synthesis proceeded with selective esterification, glycosylation ${ }^{8}$ and deacetylation, respectively. In the first step. anhydrous citric acid (1) was selectively methylated with methanolic $\mathrm{H}_{2} \mathrm{SO}_{4}$ to give 1.5-dimethyl citrate (2) in $60 \%$ yield. This sym-dimethyl citrate was further methylated with methanolic $\mathrm{H}_{2} \mathrm{SO}_{4}$ by addition of 2,2-dimethoxypropane to produce trimethylcitrate (3) in $69 \%$ yield. COSY, HMBC, HMQC and NOESY spectra proved this structure (data not shown). The direct esterification of $\mathbf{1}$ to $\mathbf{3}$ with methanolic $\mathrm{HCl}$ resulted in a poor yield $(20 \%) .{ }^{9}$ In the third step, 3 was coupled with galactose pentaacetate by using boron trifluoride diethyl etherate $\left(\mathrm{BF}_{3}-\mathrm{Et}_{2} \mathrm{O}\right)$ to produce new synthetic trimethylcitryl- $\beta$-D-tetraacetylgalatopyranoside (4) in $90 \%$ yield. The characteristic signal for anomeric proton was observed at $\delta 4.47$ (d, $J=7.7 \mathrm{~Hz}$ ), which suggested the $\beta$ configuration of a sugar unit. The positive specific rotation value $\left(+51.2^{\circ}\right)$ was consistent with the identity of the sugar unit as $\beta$ - D-galactose. Positive ion-direct chemical ionization mass spectrometry (PI-DCIMS) showed peaks at $m / z$ 582 for $\left[\mathrm{M}+\mathrm{NH}_{4}\right]^{-}$, at $m / z 366$ for [galactose tetraacetate + $\left.\mathrm{NH}_{4}\right]^{+}$, and at $m / z 252$ for [trimethylcitrate $\left.+\mathrm{NH}_{4}\right]^{+}$. Its ${ }^{3} \mathrm{H}-$ NMR and ${ }^{13} \mathrm{C}$-NMR spectral data are given in Table 1 and 2 , respectively.

In the final step, compound 4 was deacetylated by sodium methoxide followed by neutralization by passage through an Amberlite IR-120 $\left(\mathrm{H}^{+}\right)$ion exchange column ${ }^{10}$ to provide trimethylcitryl- $\beta$-D-galactopyranoside (5) as colorless crystals. Instrumental and physical analyses of the synthetic conpound were identical with the authentic natural compound previously isolated from the roots of Gastrodia elata. The

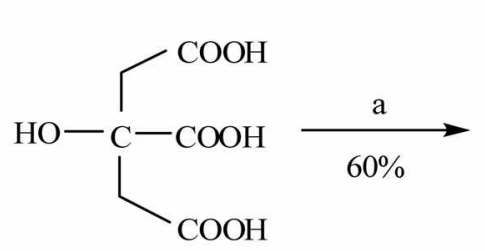

1<smiles>COC(=O)CC(O)(CC(=O)OC)C(=O)O</smiles>

2

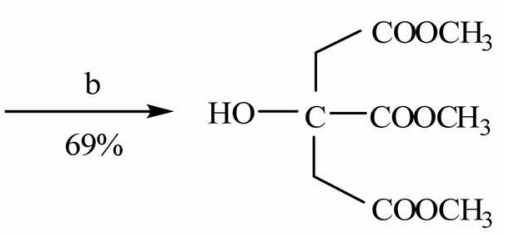

3<smiles>COC(=O)CC(CC(=O)OC)(OC(O)[C@H]1O[C@H](CO)[C@@H](O)[C@H](O)[C@H]1O)C(=O)OC</smiles>

5

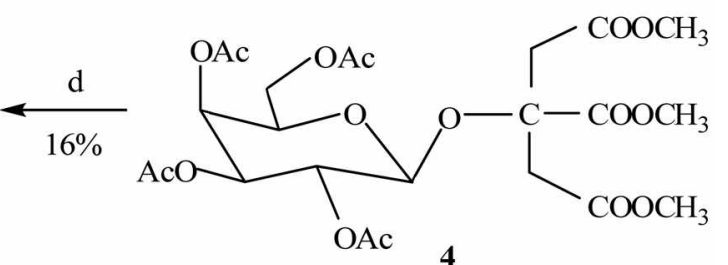

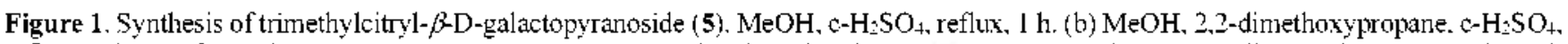
reflux. $7 \mathrm{~h}$. (c) $\beta$ D-galactose pentacetate, $\mathrm{BF}_{3}-\mathrm{Et}_{2} \mathrm{O}, \mathrm{rt} .3 \mathrm{~h}$. (d) i: $\mathrm{CH}_{3} \mathrm{ONa}$. $\mathrm{N}_{2}$ stream. rt. $1 \mathrm{~h}$. ii: neutralization by passage through Amberlite $\mathrm{R}-120$ (H) column. 
Table 1. 'H-NMR Spectral Data of Compounds 2-5 in $\mathrm{CD}_{3} \mathrm{OD}$

\begin{tabular}{|c|c|c|c|c|}
\hline $\mathrm{H}$ & 2 & 3 & 4 & 5 \\
\hline $2^{\prime \prime}$ & $2.81,2.95$ & $2.90,3.11$ & $2.75,2.98$ & 2.78 .2 .95 \\
\hline $4^{a t}$ & $2.81,2.95$ & $2.90,3.11$ & $2.75,2.98$ & 2.78 .2 .95 \\
\hline \multirow[t]{2}{*}{$\mathrm{CH}_{2}$} & $3.66,3.76$ & 3.73 (2xMe) & $3.66(2 \times \mathrm{Xe})$ & $3.65(2 \times \mathrm{Me})$ \\
\hline & & 3.85 & 3.77 & 3.76 \\
\hline $\mathrm{OA}$ & - & - & $1.98-2.17(\mathrm{~m})$ & - \\
\hline $1^{\prime}$ & - & - & $4.47(\mathrm{~d}, 7.7)$ & $4.77(\mathrm{~d}, 7.8)$ \\
\hline $2^{\prime}$ & - & - & 5.43 (dd, $9.4,7.7$ ) & 3.65 (dd, $9.8,7.6$ ) \\
\hline $3^{\prime}$ & - & - & 5.33 (dd, $9.4,7.7)$ & $3.51(\mathrm{dd}, 9.6,3.2)$ \\
\hline $4^{\prime}$ & - & - & 5.25 (dd, $9.4,7.7)$ & $3.74(\mathrm{~d}, 2.4)$ \\
\hline $5^{\prime}$ & - & - & $3.35(\mathrm{~m})$ & $3.55(\mathrm{~m})$ \\
\hline \multirow[t]{2}{*}{$6^{1}$} & - & - & 4.07 (dd, $12.2,2.5)$ & 3.71 (dd, $11,6,4,4)$ \\
\hline & & & 4.14 (dd, $12.2,4.6)$ & $3.82(\mathrm{dd}, 11,6,7,6)$ \\
\hline
\end{tabular}

"AB system. $J=15.6 \mathrm{~Hz}$

Table 2. 'S-NMR Spectral Data of Compounds 2-5 in $\mathrm{CD}_{3} \mathrm{OD}$

\begin{tabular}{|c|c|c|c|c|}
\hline C & 2 & 3 & 4 & 5 \\
\hline 1 & 170.8 & 172.1 & 171.5 & 170.9 \\
\hline 2 & 43.5 & 43.8 & 44.2 & 43.5 \\
\hline 3 & 73.4 & 74.0 & 74.6 & 74.2 \\
\hline 4 & 43.5 & 43.8 & 44.2 & 43.5 \\
\hline 5 & 170.8 & 172.1 & 171.5 & 170.9 \\
\hline \multirow[t]{2}{*}{$\mathrm{CH}_{3}$} & 51.5 & $52.7(2 \times \mathrm{Me})$ & $52.2(2 \mathrm{xMe})$ & $51.4(2 \mathrm{xMe})$ \\
\hline & 51.5 & 53.5 & 53.1 & 52.3 \\
\hline $\mathrm{COO}$ & 175.4 & 175.4 & 171.8 & 171.2 \\
\hline Acetyl-C.H & - & - & 20.5 & - \\
\hline Acetyl-Co & - & - & 175.0 & - \\
\hline$l^{\prime}$ & - & - & 96.3 & 97.9 \\
\hline $2^{\prime}$ & - & - & 72.0 & 70.8 \\
\hline $3^{\prime}$ & - & - & 72.6 & 73.0 \\
\hline $4^{\prime}$ & - & - & 70.1 & 70.4 \\
\hline $5^{\prime}$ & - & - & 74.5 & 75.8 \\
\hline $6^{\prime}$ & - & - & 62.9 & 61.9 \\
\hline
\end{tabular}

final step of the reaction, however, gave a relatively poor yield $(16 \%)$. The major product of this deprotection step was, unexpectedly, trimethylcitrate (3), which can be produced by nucleophilic attack of sodium methoxide to the glycosidic linkage. Deacetylation of peracetylated glycosides under acidic conditions leads to the cleavage of the glycosidic bond. ${ }^{1}$

In conclusion, we were successful in synthesizing natural trimethylcitryl- $\beta$-D-galactopyranoside (5), starting from citric acid in a four-step reaction.

\section{Experimental Section}

General. Melting point was measured on an Electrothermal IA9100 apparatus (Thermo Scientific, Pittsburgh, PA, USA) and are uncorrected. NMR spectra were recorded on a UNITY-500 or GEMNI-200 spectrophotometer (Varian, Palo Alto, CA, USA) using $\mathrm{CD}_{3} \mathrm{OD}$ as a solvent. PI-DCIMS spectra were measured with a Model MAT95 or LCQ mass spectrometer (Thermo Scientific) and fast atom bombard- ment (FAB) mass spectra were acquired with a JMS 700 mass spectrometer (Jeol, Tokyo, Japan). Specific rotation was measured on a DIP-370 digital polarimeter (Jasco, Easton, MD, USA). Thin layer chromatography utilized a Kieselgel $60 \mathrm{~F}_{25+4}$ plate $(0.1 \mathrm{~mm}$; Merck, Darmstadt, Germany) using a solvent system of 1,2-dichloroethane:methanol: formic acid $(7: 3: 0.5)$, a spray consisting of $0.1 \%$ alcoholic bromocresol green, then coloration in an iodine chamber for citric acid derivatives. For glycosides, a solvent system consisting of $n$-butanol:acetic acid:diethylether:water $(9: 6: 3: 1)$ and detection by spraying with diphenylamine reagent followed by heat-mediated coloration were used. Citric acid monohydrate, boron trifluoride diethyl etherate, $\beta$-D-galactose pentaacetate, 2,2-dimethoxypropane, and Amberlite IR$120\left(\mathrm{H}^{+}\right)$ion exchange resin were all purchased from SigmaAldrich (St. Louis, MO, USA).

Preparation of 1,5-dimethylcitrate (2). Commercial citric acid monohydrate was dried for $24 \mathrm{~h}$ under reduced pressure at $85^{\circ} \mathrm{C}$ to give anhydrous citric acid (1). Compound $1(34.6 \mathrm{~g}, 0.18 \mathrm{~mol})$ in methanol $(200 \mathrm{~mL})$ and $\mathrm{c}$ $\mathrm{H}_{2} \mathrm{SO}_{4}(1 \mathrm{~mL})$ was refluxed for $1 \mathrm{~h}$. The reaction mixture was diluted with water and neutralized with $1 \mathrm{~N} \mathrm{YaOH}$ to generate a clear solution $(\mathrm{pH} 7.0)$, which was thoroughly concentrated in vacuo. The residue was suspended in acetone and filtered to remove by-products. After the filtrate was concentrated and suspended with water, c-HCl was added slowly in an ice bath, followed by stirring for $10 \mathrm{~min}$. The precipitate was collected and washed with water, then recrystallized with $30 \% \mathrm{MeOH}$ to yield compound 2 ( $23.8 \mathrm{~g}$, $60 \%$ yield) as colorless amorphous crystals. $\mathrm{Rf}$ value: 0.80 . mp $109-115^{\circ} \mathrm{C}\left(116-121^{\circ} \mathrm{C},{ }^{12} 122-124^{\circ} \mathrm{C}^{13}\right)$. FT-IR (nujol) $\mathrm{cm}^{-1}: 3476(\mathrm{OH}), 1742$ (ester); PI-DCIMS m/z: $221[\mathrm{M}+$ $\mathrm{H}]^{+}$; ${ }^{1} \mathrm{H}$ - and ${ }^{13} \mathrm{C}-\mathrm{NMR}$ data: see Table 1 and Table 2 , respectively. The $\mathrm{OH}$ signal ( $\delta 4.88 \mathrm{ppm})$ in $\mathrm{COOH}$ group could be assigned after $\mathrm{D}_{2} \mathrm{O}$ exchange.

Preparation of trimethylcitrate (3). Compound 2 (15.6 $\mathrm{g}, 0.07 \mathrm{~mol}$ ) in $\mathrm{MeOH}(190 \mathrm{~mL}$ ), 2,2-dimethoxypropane (10 $\mathrm{ml})$ and $\mathrm{c}_{-} \mathrm{H}_{2} \mathrm{SO}_{4}(1.25 \mathrm{~mL})$ was refluxed for $7 \mathrm{~h}$. After the reaction mixture was thoroughly concentrated in vacuo, the remaining oily material was crystallized with $30 \% \mathrm{MeOH}$ to afford compound 3 ( $11.3 \mathrm{~g}, 69 \%$ yield) as colorless amorphous crystals. Rf value: $0.88 . \operatorname{mp} 72-76^{\circ} \mathrm{C}\left(76^{\circ} \mathrm{C}^{l-1}\right)$. FT-IR (nujol) $\mathrm{cm}^{-1}: 3486(\mathrm{OH}), 1756$ (ester); PI-DCIMS $m / z: 235$ $[\mathrm{M}+\mathrm{H}]^{+}, 252\left[\mathrm{M}+\mathrm{NH}_{4}\right]^{+} ;{ }^{1} \mathrm{H}$ - and ${ }^{13} \mathrm{C}-\mathrm{NMR}$ data: see Table 1 and Table 2, respectively. The $\mathrm{OH}$ proton $(\delta 1.32$ ppm) almost disappeared after $\mathrm{D}_{2} \mathrm{O}$ exchange.

Synthesis of trimethylcitryl- $\beta$-D-tetraacetylgalatopyranoside (4). $\mathrm{BF}_{3}-\mathrm{Et}_{2} \mathrm{O}(1.42 \mathrm{~g}, 0.01 \mathrm{~mol})$ was added dropwise to the mixture of $\beta$-D-galactose pentaacetate $(3.9 \mathrm{~g}, 0.01 \mathrm{~mol})$, methylene chloride $(60 \mathrm{~mL})$ and trimethylcitrate $(2.34 \mathrm{~g}$, $0.01 \mathrm{~mol}$ ). The mixture was kept in the dark using aluminium foil and stirred for $3 \mathrm{~h}$ at room temperature. Excess $\mathrm{BF}_{3-}$ $\mathrm{Et}_{2} \mathrm{O}$ was decomposed with a saturated $\mathrm{NaHCO}_{3}$ solution. After dilution with methylene chloride, the mixture was washed with water, dried and concentrated to produce compound 4 (5.08 g, 90\%) as a colorless oily material. Rf value: 0.71. $[\alpha]_{\mathrm{D}}+51.2^{\circ}\left(\mathrm{c}=0.363, \mathrm{CH}_{3} \mathrm{OH}\right)$; IR (nujol) $\mathrm{cm}^{-1}: 1753$ 
(ester), 1739 (acetyl); PI-DCIMS $m / z: 582\left[\mathrm{M}+\mathrm{NH}_{4}\right]^{+}, 366$ [galactose tetraacetate $\left.+\mathrm{NH}_{4}\right]^{-}, 252$ [trimethylcitrate $\left.+\mathrm{NH}_{4}\right]^{-}$; ${ }^{1} \mathrm{H}$ - and ${ }^{13} \mathrm{C}-\mathrm{NMR}$ data: see Table 1 and Table 2 , respectively).

Synthesis of trimethylcitryl- $\beta$-D-galactopyranoside (5). Compound 4 (175.4 mg. $0.31 \mathrm{mmol}$ ) was dissolved in anhydrous $\mathrm{MeOH}(5 \mathrm{~mL})$ under a nitrogen atmosphere. Freshly prepared $0.1 \times \mathrm{CH}_{3} \mathrm{ONa}(0.2 \mathrm{~mL})$ was added dropwise to this solution under a nitrogen stream and stirred for 1 $\mathrm{h}$ at room temperature. After the reaction mixture was neutralized by passage through an Amberlite IR-120 $\left(\mathrm{H}^{+}\right)$ ion exchange column, the collected solution was concentrated to furnish a colorless oily material. The crystallization of this material with $\mathrm{MeOH}$ yielded amorphous crystals. which were identified as trimethylcitrate (3). From the mother liquid compound 5 (20 mg, 16\%) was obtained as colorless crystals. Rf value: 0.27 mp: $136-139{ }^{\circ} \mathrm{C}$; $[\alpha]_{\mathrm{D}}$ $+22.8^{\circ}$ (c $=0.309, \mathrm{CH}_{3} \mathrm{OH}$ ); IR (nujol) $\mathrm{cm}^{-1}: 3480(\mathrm{OH})$, 1753 (ester); FAB-MS $m / z: 419$ [M+Na] ${ }^{-}, 235$ [trimethylcitrate $+\mathrm{H}]^{+}$(base peak); ${ }^{1} \mathrm{H}$ - and ${ }^{13} \mathrm{C}-\mathrm{\lambda} \mathrm{MR}$ data: see Table 1 and Table 2, respectively).

Acknowledgments. This work was supported by grant No. R05-2002-000-00682-0 from the Basic Research Program of the Korea Science \& Engineering Foundation.

\section{References}

1. Tang, W; Eisenbrand, G. Chinese Dntgs of Plan Origin. Chemist Phannacolog; and Use in Traditional and Modern Medicine; Springer Verlag: Berlin Heidelberg, Germany, 1992; pp 545-547.

2. Taguchi, H.; Yosioka, 1.; Yamasaki, K.; Kim, I. H. Chem. Pham. Butl. 1981, 29,55.

3. Zhou, J.; Yang, Y. B.; Pu, X. Y. Yimun Chih Wi Yen Chin 1908, 2,370

4. Yun-Choi, H. S.; Pyo, M. K.; Park, K. M. Arch. Pharm. Res. 1998. $21,357$.

5. Lin, J. H.; Liu, Y. C.; Hau, J. P.; Wen, K. C. Phrochem. 1996, 42 , 549.

6. Pyo, M. K.; Park, K. M.; Yun-Choi, H. S. Not. Prod. Sci, 2000, 6, 53.

7. Choi, J. H.; Lee, D. U. Chem. Phom. Bmll. 2006, 54, 1720.

8. Lee, B. Y.; Baek, J. Y.; Jeon, H. B.; Kim, K. D. Bull. Kor: Chem. Soc. $2007,28,257$.

9. Saleem, R.; Ahmad, M.; Naz, A.; Siddiqui, H.; Ahmad, S. I. Arch. Phown. Res. 2004, 27, 1037.

10. Kartal, S.; Tokalioglu, S.; Ozkan, B. Bull. Kor: Chem. Soc. 2006, 27,694 .

11. Emmerie, A.; Engel, C. Rec. Tran Chim Pavs-Bas. 1939, 58 , 895 .

12. Miyake, M.; Kamimoto, Y.; Soráchi, M. Biochim. Biophis. Acta $1978,544,656$.

13. Hirota, K.; Kitagawa, H.; Shimamura, M.; Ohnori, S. Chem. Lett. 1980, 191

14. Anet, F. A. L.; Park, J. M. J. Am. Chem. Soc. 1992, $114,411$. 